\title{
Inequalities for $\mathbb{B}$-convex functions via generalized fractional integral
}

Ilknur Yesilce ${ }^{1 *}$ (D)

\section{"Correspondence:}

ilknuryesilce@gmail.com

${ }^{1}$ Faculty of Science and Letters,

Aksaray University, Aksaray, Turkey

\begin{abstract}
Recently, fractional calculus has become a very popular and important area. Specially, fractional integral inequalities have been studied by different authors. In this article, we give new Hermite-Hadamard type inequalities for $\mathbb{B}$-convex functions via Riemann-Liouville and Hadamard fractional integrals. Also, we show that the inequalities involve the fractional integrals of a function with respect to the function $g$ which are the more general form of these obtained Hermite-Hadamard inequalities.
\end{abstract}

MSC: 26A33; 52A40; 52A41

Keywords: Generalized fractional integral; Hermite-Hadamard inequality; $\mathbb{B}$-convex functions; Abstract convexity

\section{Introduction}

The idea of fractional calculus was suggested by Leibnitz via a letter to L'Hospital. Then, a lot of famous scientists studied this notion in different areas like inequality theory, applied mathematics, engineering [10,12, 13, 15, 21, 26, 30, 31, 33] (see also [29] for the historical considerations). Recently, the importance of fractional calculus can be more understandable, and numerous works involving fractional calculus have been done. Specially, for integral inequalities, which is the topic of this work, fractional integrals have been used, thus familiar significant inequalities can be generalized using this type of integrals. Hermite-Hadamard inequality, which is one of these types of inequalities and one of important applications of convexity, was introduced in $[19,20]$. Additionally, a lot of authors have examined different generalizations of the convexity $[4,6-8,22,34]$. Then, it has been studied for different abstract convex functions [1-3, 5, 17, 18, 27, 28, 35]. Recently, these works have been given with a new generalized form of inequalities via fractional integrals $[11,14,32,36]$.

$\mathbb{B}$-convexity was introduced in [16]. Then, $\mathbb{B}$-convex sets, $\mathbb{B}$-convex functions, separation theorems, and application to mathematical economy were analyzed $[4,16,23]$.

In this article, Hermite-Hadamard inequality is worked for $\mathbb{B}$-convex functions. The classic format of Hermite-Hadamard inequality for $\mathbb{B}$-convex functions was proved in [37]. For $\mathbb{B}$-convex functions, Riemann-Liouville fractional Hermite-Hadamard inequality was studied in [36]. Inequalities given in this paper differ from previous inequalities in using generalized fractional integral. Thus, the most general form of Hermite-Hadamard inequality for $\mathbb{B}$-convex functions is obtained.

(c) The Author(s) 2019. This article is distributed under the terms of the Creative Commons Attribution 4.0 International License (http://creativecommons.org/licenses/by/4.0/), which permits unrestricted use, distribution, and reproduction in any medium, provided you give appropriate credit to the original author(s) and the source, provide a link to the Creative Commons license, and indicate if changes were made. 
We can point out the aim of the paper as follows. The most general HermiteHadamard inequality for $\mathbb{B}$-convex functions is proven, then we give the relation between this inequality and the previous inequalities which contain the Riemann-Liouville and Hadamard fractional integrals. Thus, it shows that the final inequality presented in the article is the generalization of the others as well as the classic one for $\mathbb{B}$-convex functions.

In the second section, we recall definitions of different types fractional integrals. Additionally, we give some required definitions and theorems about $\mathbb{B}$-convexity and former inequality for $\mathbb{B}$-convex functions. In the next section, we show inequalities involving Riemann-Liouville fractional integral. In the fourth section, we introduce HermiteHadamard type inequalities via Hadamard fractional integrals. In the last section, we prove Hermite-Hadamard inequalities for $\mathbb{B}$-convex functions via fractional integrals of a function with respect to the function $g$. Also, we show that the Hermite-Hadamard inequalities involving Riemann-Liouville fractional integral and Hadamard fractional integral can be obtained as our final inequalities.

\subsection{Fractional integral types}

Let us recall the following definitions of fractional integral types. Throughout the paper, let $f:[a, b] \rightarrow \mathbb{R}$ be a given function, where $0 \leq a<b<+\infty$ and $f \in L_{1}[a, b]$. Also, $\Gamma(\alpha)$ is the gamma function.

Definition 1 ([24]) The left-sided Riemann-Liouville integral $J_{a+}^{\alpha} f$ and the right-sided Riemann-Liouville integral $J_{b}^{\alpha} f$ of order $\alpha>0$ with $a \geq 0$ are defined by

$$
J_{a+}^{\alpha} f(x)=\frac{1}{\Gamma(\alpha)} \int_{a}^{x}(x-t)^{\alpha-1} f(t) d t, \quad x>a,
$$

and

$$
J_{b-}^{\alpha} f(x)=\frac{1}{\Gamma(\alpha)} \int_{x}^{b}(t-x)^{\alpha-1} f(t) d t, \quad x<b,
$$

respectively.

Definition $2([24,25])$ The left-sided Hadamard fractional integral $\mathbf{J}_{a+}^{\alpha}$ of order $\alpha>0$ of $f$ is defined by

$$
\mathbf{J}_{a+}^{\alpha} f(x)=\frac{1}{\Gamma(\alpha)} \int_{a}^{x}\left(\ln \frac{x}{t}\right)^{\alpha-1} \frac{f(t)}{t} d t, \quad x>a,
$$

provided that the integral exists. The right-sided Hadamard fractional integral $\mathbf{J}_{b-}^{\alpha}$ of order $\alpha>0$ of $f$ is defined by

$$
\mathbf{J}_{b-}^{\alpha} f(x)=\frac{1}{\Gamma(\alpha)} \int_{x}^{b}\left(\ln \frac{t}{x}\right)^{\alpha-1} \frac{f(t)}{t} d t, \quad x<b,
$$

provided that the integral exists.

Definition 3 ([24]) Let $g:[a, b] \rightarrow \mathbb{R}$ be an increasing and positive monotone function on $(a, b]$ having a continuous derivative $g^{\prime}(x)$ on $(a, b)$. The left-sided fractional integral of 
$f$ with respect to the function $g$ on $[a, b]$ of order $\alpha>0$ is defined by

$$
I_{a^{+} ; g}^{\alpha} f(x)=\frac{1}{\Gamma(\alpha)} \int_{a}^{x} \frac{g^{\prime}(t) f(t)}{[g(x)-g(t)]^{1-\alpha}} d t, \quad x>a,
$$

provided that the integral exists. The right-sided fractional integral of $f$ with respect to the function $g$ on $[a, b]$ of order $\alpha>0$ is defined by

$$
I_{b^{-} ; g}^{\alpha} f(x)=\frac{1}{\Gamma(\alpha)} \int_{x}^{b} \frac{g^{\prime}(t) f(t)}{[g(t)-g(x)]^{1-\alpha}} d t, \quad x<b,
$$

provided that the integral exists.

\section{$1.2 \mathbb{B}$-Convexity}

Let $r \in \mathbb{N}, \varphi_{r}: \mathbb{R} \rightarrow \mathbb{R}, \varphi_{r}(x)=x^{2 r+1}$ and $\Phi_{r}: \mathbb{R}^{n} \rightarrow \mathbb{R}^{n}, \Phi_{r}(\boldsymbol{x})=\Phi_{r}\left(x_{1}, x_{2}, \ldots, x_{n}\right)=$ $\left(\varphi_{r}\left(x_{1}\right), \varphi_{r}\left(x_{2}\right), \ldots, \varphi_{r}\left(x_{n}\right)\right)$. For a finite nonempty set $A=\left\{\boldsymbol{x}^{(1)}, \boldsymbol{x}^{(2)}, \ldots, \boldsymbol{x}^{(m)}\right\} \subset \mathbb{R}^{n}$, the $\mathrm{r}-$ convex hull of $A$, denoted as $\operatorname{Co}^{r}(A)$, is given by

$$
\operatorname{Co}^{r}(A)=\left\{\Phi_{r}^{-1}\left(\sum_{i=1}^{m} t_{i} \Phi_{r}\left(\boldsymbol{x}^{(i)}\right)\right): t_{i} \geq 0, \sum_{i=1}^{m} t_{i}=1\right\} .
$$

Definition 4 ([16]) The Kuratowski-Painleve upper limit of the sequence of sets $\left(\mathrm{Co}^{r}(A)\right)_{r \in \mathbb{N}}$, denoted by $\mathrm{Co}^{\infty}(A)$ where $A$ is a finite subset of $\mathbb{R}^{n}$, is called $\mathbb{B}$-polytope of $A$.

Definition 5 ([16]) A subset $U$ of $\mathbb{R}^{n}$ is $\mathbb{B}$-convex if for all finite subsets $A \subset U$ the $\mathbb{B}$ polytope $\mathrm{Co}^{\infty}(A)$ is contained in $U$.

In $\mathbb{R}_{+}^{n}$, a $\mathbb{B}$-convex set is defined in a different way [16]:

A subset $U$ of $\mathbb{R}_{+}^{n}$ is $\mathbb{B}$-convex if and only if, for all $\boldsymbol{x}^{(1)}, \boldsymbol{x}^{(2)} \in U$ and all $\lambda \in[0,1]$, one has $\lambda \boldsymbol{x}^{(1)} \vee \boldsymbol{x}^{(2)} \in U$.

Here, we denote the least upper bound with respect to the coordinate-wise order relation of $\boldsymbol{x}^{(1)}, \boldsymbol{x}^{(2)}, \ldots, \boldsymbol{x}^{(m)} \in \mathbb{R}^{n}$ by $\bigvee_{i=1}^{m} \boldsymbol{x}^{(i)}$, that is,

$$
\bigvee_{i=1}^{m} \boldsymbol{x}^{(i)}=\left(\max \left\{x_{1}^{(1)}, x_{1}^{(2)}, \ldots, x_{1}^{(m)}\right\}, \ldots, \max \left\{x_{n}^{(1)}, x_{n}^{(2)}, \ldots, x_{n}^{(m)}\right\}\right)
$$

where $x_{j}^{(i)}$ denotes $j$ th coordinate of the point $\boldsymbol{x}^{(i)}$.

Remark 1 In $\mathbb{R}_{+}, \mathbb{B}$-convex sets are intervals because of definition. Indeed, for all $x, y \in \mathbb{R}_{+}$ and $\lambda \in[0,1]$, all the combinations of $\lambda x \vee y$ are either an interval of $[y, x]$ or the point $y$ depends on the order of $x$ and $y$. Since all the elements of a $\mathbb{B}$-convex set satisfy this condition, a $\mathbb{B}$-convex subset of $\mathbb{R}_{+}$is an interval.

Furthermore, in $[16,23]$, the definition of $\mathbb{B}$-convex functions is given as follows.

Definition $6([16,23])$ Let $U \subset \mathbb{R}^{n}$. A function $f: U \rightarrow \mathbb{R} \cup\{ \pm \infty\}$ is called a $\mathbb{B}$-convex function if $\operatorname{epi}(f)=\{(\boldsymbol{x}, \mu) \mid \boldsymbol{x} \in U, \mu \in \mathbb{R}, \mu \geq f(\boldsymbol{x})\}$ is a $\mathbb{B}$-convex set. 
The following theorem provides a sufficient and necessary condition for $\mathbb{B}$-convex functions in $\mathbb{R}_{+}^{n}$.

Theorem $1([16,23])$ Let $U \subset \mathbb{R}_{+}^{n}, f: U \rightarrow \mathbb{R}_{+} \cup\{+\infty\}$. The function $f$ is $\mathbb{B}$-convex if and only if $U$ is a $\mathbb{B}$-convex set, and for all $\mathbf{x}, \boldsymbol{y} \in U$ and all $\lambda \in[0,1]$, the following inequality holds:

$$
f(\lambda \mathbf{x} \vee \boldsymbol{y}) \leq \lambda f(\mathbf{x}) \vee f(\boldsymbol{y}) .
$$

\subsection{Hermite-Hadamard inequality for $\mathbb{B}$-convex functions}

Hermite-Hadamard inequality for $\mathbb{B}$-convex functions was given with the following theorem in [37].

Theorem 2 ([37]) Let $f:[a, b] \subset \mathbb{R}_{+} \rightarrow \mathbb{R}_{+}$be a $\mathbb{B}$-convex function. Then one has the inequality

$$
\frac{1}{b-a} \int_{a}^{b} f(t) d t \leq \begin{cases}f(a), & 1 \leq \frac{f(a)}{f(b)}, \\ \frac{b\left(\left[(f(a)]^{2}+[(b)]\right]^{2}\right)-2 a f(a) f(b)}{2(b-a) f(b)}, & 0 \leq \frac{f(a)}{f(b)}<1 .\end{cases}
$$

\section{Results and discussion}

\subsection{Hermite-Hadamard type inequalities involving Riemann-Liouville fractional integral}

We proved the Riemann-Liouville fractional Hermite-Hadamard inequalities for $\mathbb{B}$ convex functions which were given in the following theorems for left-sided integral and right-sided integral, respectively, in [36].

Theorem 3 ([36]) Let $f:[a, b] \subset \mathbb{R}_{+} \rightarrow \mathbb{R}_{+}$and $f \in L_{1}[a, b]$. If $f$ is a $\mathbb{B}$-convex function on $[a, b]$, then the following inequality for fractional integrals holds:

$$
J_{a+}^{\alpha} f(b) \leq \begin{cases}\frac{f(a)(b-a)^{\alpha}}{\Gamma(\alpha+1)}, & 1 \leq \frac{f(a)}{f(b)} \\ \frac{b^{\alpha}(f(b)-f(a))^{\alpha+1}+(\alpha+1) f(a)(f(b))^{\alpha}(b-a)^{\alpha}}{\Gamma(\alpha+2)(f(b))^{\alpha}}, & 0 \leq \frac{f(a)}{f(b)}<1\end{cases}
$$

with $\alpha>0$.

Theorem 4 ([36]) Let $f:[a, b] \subset \mathbb{R}_{+} \rightarrow \mathbb{R}_{+}$and $f \in L_{1}[a, b]$. Iff is a $\mathbb{B}$-convex function on $[a, b]$, then the following inequality for fractional integrals holds:

$$
J_{b-}^{\alpha} f(a) \leq \begin{cases}\frac{f(a)(b-a)^{\alpha}}{\Gamma(\alpha+1)}, & 1 \leq \frac{f(a)}{f(b)}, \\ \frac{(b f(a)-a f(b))^{\alpha+1}+(f(b))^{\alpha+1}(b-a)^{\alpha}(\alpha b+a)}{b \Gamma(\alpha+2)(f(b))^{\alpha}}, & \frac{a}{b} \leq \frac{f(a)}{f(b)}<1, \\ \frac{f(b)(b-a)^{\alpha}(a b+a)}{b \Gamma(\alpha+2)}, & 0 \leq \frac{f(a)}{f(b)}<\frac{a}{b}\end{cases}
$$

with $\alpha>0$.

In Theorem 3 and Theorem 4, the inequalities were obtained by getting the multiplier $(1-\lambda)^{\alpha-1}$ and $\left(\lambda-\frac{a}{b}\right)^{\alpha-1}$. We derive nearly the same inequalities with changing the multipliers. For example, if we take the multiplier $\frac{(\max \{a, \lambda b\})^{\prime}}{[b-\max \{a, \lambda b\}]^{1-\alpha}}$ for left-sided integral, we deduce 
inequality (11) and the same inequality given in (10) to take the multiplier $\frac{(\max \{a, \lambda b\})^{\prime}}{[\lambda b-a]^{1-\alpha}}$ for the right-sided integral.

Theorem 5 Let $f:[a, b] \subset \mathbb{R}_{+} \rightarrow \mathbb{R}_{+}$and $f \in L_{1}[a, b]$. Iff is a $\mathbb{B}$-convex function on $[a, b]$, then the following inequality holds:

$$
J_{a+}^{\alpha} f(b) \leq \begin{cases}\frac{f(a)(b-a)^{\alpha}}{\Gamma(\alpha+1)}, & 1 \leq \frac{f(a)}{f(b)} \\ \frac{b^{\alpha}(f(b)-f(a))^{\alpha+1}+(\alpha+1) f(a)(f(b))^{\alpha}(b-a)^{\alpha}}{\Gamma(\alpha+2)(f(b))^{\alpha}}, & \frac{a}{b} \leq \frac{f(a)}{f(b)}<1, \\ \frac{f(b)(b-a)^{\alpha}(\alpha a+b)}{b \Gamma(\alpha+2)}, & 0 \leq \frac{f(a)}{f(b)}<\frac{a}{b}\end{cases}
$$

with $\alpha>0$.

Proof Let $f$ be a $\mathbb{B}$-convex function. Thus, inequality (7) holds. For desired inequality, we will multiply both sides of inequality $(7)$ by $\frac{(\max \{a, \lambda b\})^{\prime}}{[b-\max \{a, \lambda b\}]^{1-\alpha}}$ and then integrate with respect to $\lambda$ over $[0,1]$. For the left-hand side of inequality, we get that

$$
\begin{aligned}
& \int_{0}^{1} \frac{(\max \{a, \lambda b\})^{\prime}}{[b-\max \{a, \lambda b\}]^{1-\alpha}} f(\max \{a, \lambda b\}) d \lambda \\
& \quad=\int_{0}^{\frac{a}{b}} \frac{(a)^{\prime}}{[b-a]^{1-\alpha}} f(a) d \lambda+\int_{\frac{a}{b}}^{1} \frac{(\lambda b)^{\prime}}{[b-\lambda b]^{1-\alpha}} f(\lambda b) d \lambda \\
& \quad=\int_{a}^{b} \frac{f(t)}{[b-t]^{1-\alpha}} d t=\Gamma(\alpha) J_{a^{+}}^{\alpha} f(b) .
\end{aligned}
$$

For the right-hand side of inequality, we have to examine two cases of $1 \leq \frac{f(a)}{f(b)}$ and $0 \leq$ $\frac{f(a)}{f(b)}<1$. For the first case, we have

$$
\begin{aligned}
& \int_{0}^{1} \frac{(\max \{a, \lambda b\})^{\prime}}{[b-\max \{a, \lambda b\}]^{1-\alpha}} \max \{f(a), \lambda f(b)\} d \lambda \\
& \quad=\int_{0}^{1} \frac{(\max \{a, \lambda b\})^{\prime}}{[b-\max \{a, \lambda b\}]^{1-\alpha}} f(a) d \lambda \\
& \quad=f(a)\left[\int_{0}^{\frac{a}{b}} \frac{(a)^{\prime}}{[b-a]^{1-\alpha}} d \lambda+\int_{\frac{a}{b}}^{1} \frac{(\lambda b)^{\prime}}{[b-\lambda b]^{1-\alpha}} d \lambda\right] \\
& \quad=f(a) b^{\alpha} \int_{\frac{a}{b}}^{1}[1-\lambda]^{\alpha-1} d \lambda=\frac{f(a)(b-a)^{\alpha}}{\alpha} .
\end{aligned}
$$

Thus the inequality is

$$
J_{a^{+}}^{\alpha} f(b) \leq \frac{f(a)(b-a)^{\alpha}}{\Gamma(\alpha+1)}
$$

For the second case, it can be

(i) $\frac{a}{b} \leq \frac{f(a)}{f(b)}<1$ or

(ii) $0 \leq \frac{f(a)}{f(b)}<\frac{a}{b}$. 
Let us examine (i). If $\frac{a}{b} \leq \frac{f(a)}{f(b)}<1$, then the following equality is obtained:

$$
\begin{aligned}
& \int_{0}^{1} \frac{(\max \{a, \lambda b\})^{\prime}}{[b-\max \{a, \lambda b\}]^{1-\alpha}} \max \{f(a), \lambda f(b)\} d \lambda \\
& \quad=\int_{0}^{\frac{a}{b}} \frac{(a)^{\prime}}{[b-a]^{1-\alpha}} f(a) d \lambda+\int_{\frac{a}{b}}^{\frac{f(a)}{f(b)}} \frac{(\lambda b)^{\prime}}{[b-\lambda b]^{1-\alpha}} f(a) d \lambda+\int_{\frac{f(a)}{f(b)}}^{1} \frac{(\lambda b)^{\prime}}{[b-\lambda b]^{1-\alpha}} \lambda f(b) d \lambda \\
& \quad=b^{\alpha} f(a) \int_{\frac{a}{b}}^{\frac{f(a)}{f(b)}}[1-\lambda]^{\alpha-1} d \lambda+b^{\alpha} f(b) \int_{\frac{f(a)}{f(b)}}^{1} \lambda[1-\lambda]^{\alpha-1} d \lambda \\
& \quad=\frac{b^{\alpha}(f(b)-f(a))^{\alpha+1}+(\alpha+1) f(a)(f(b))^{\alpha}(b-a)^{\alpha}}{\alpha(\alpha+1)(f(b))^{\alpha}} .
\end{aligned}
$$

Hence, we obtain the inequality

$$
J_{a^{+}}^{\alpha} f(b) \leq \frac{b^{\alpha}(f(b)-f(a))^{\alpha+1}+(\alpha+1) f(a)(f(b))^{\alpha}(b-a)^{\alpha}}{\Gamma(\alpha+2)(f(b))^{\alpha}}
$$

Let us introduce (ii). For the case of $0 \leq \frac{f(a)}{f(b)}<\frac{a}{b}$, we have

$$
\begin{aligned}
& \int_{0}^{1} \frac{(\max \{a, \lambda b\})^{\prime}}{[b-\max \{a, \lambda b\}]^{1-\alpha}} \max \{f(a), \lambda f(b)\} d \lambda \\
& \quad=\int_{0}^{\frac{f(a)}{f(b)}} \frac{(a)^{\prime}}{[b-a]^{1-\alpha}} f(a) d \lambda+\int_{\frac{f(a)}{f(b)}}^{\frac{a}{b}} \frac{(a)^{\prime}}{[b-a]^{1-\alpha}} \lambda f(b) d \lambda+\int_{\frac{a}{b}}^{1} \frac{(\lambda b)^{\prime}}{[b-\lambda b]^{1-\alpha}} \lambda f(b) d \lambda \\
& \quad=b^{\alpha} f(b) \int_{\frac{a}{b}}^{1} \lambda[1-\lambda]^{\alpha-1} d \lambda=\frac{f(b)(b-a)^{\alpha}(\alpha a+b)}{b \alpha(\alpha+1)} .
\end{aligned}
$$

Then we deduce that

$$
J_{a^{+}}^{\alpha} f(b) \leq \frac{f(b)(b-a)^{\alpha}(\alpha a+b)}{b \Gamma(\alpha+2)} .
$$

From (12), (13), and (14), we get the desired inequality.

For the right-sided integral, the inequality can be proved with the same method of the above theorem by using the multiplier $\frac{(\max \{a, \lambda b\})^{\prime}}{[\lambda b-a]^{1-\alpha}}$.

\subsection{Hermite-Hadamard type inequalities involving Hadamard fractional integral}

In this section, we introduce Hermite-Hadamard type inequalities via Hadamard fractional integral.

Theorem 6 Let $\alpha>0$. Iff is a $\mathbb{B}$-convex function on $[a, b]$, then

$$
\mathbf{J}_{a^{+}}^{\alpha} f(b) \leq \begin{cases}\frac{f(a)\left(\ln \frac{b}{a}\right)^{\alpha}}{\Gamma(\alpha+1)}, & 1 \leq \frac{f(a)}{f(b)} \\ \frac{f(a)\left[\left(\ln \frac{b}{a}\right)^{\alpha}-\left(\ln \frac{f(b)}{f(a)}\right)^{\alpha}\right]}{\Gamma(\alpha+1)}+\frac{f(b)}{\Gamma(\alpha)} \int_{\frac{f(a)}{f(b)}}^{1}\left(\ln \frac{1}{\lambda}\right)^{(\alpha-1)} d \lambda, & \frac{a}{b} \leq \frac{f(a)}{f(b)}<1, \\ \frac{f(b)}{\Gamma(\alpha)} \int_{\frac{a}{b}}^{1}\left(\ln \frac{1}{\lambda}\right)^{(\alpha-1)} d \lambda, & 0 \leq \frac{f(a)}{f(b)}<\frac{a}{b}\end{cases}
$$


Proof Let us multiply inequality (7) that comes from $\mathbb{B}$-convexity of $f$ by $(\max \{a, \lambda b\})^{\prime}$, then integrate with respect to $\lambda$ over $[0,1]$. Hereby, for the left$\max \{a, \lambda b\}[\ln b-\ln (\max \{a, \lambda b\})]^{1-\alpha}$
hand side of inequality, we have that

$$
\begin{aligned}
& \int_{0}^{1} \frac{(\max \{a, \lambda b\})^{\prime}}{\max \{a, \lambda b\}[\ln b-\ln (\max \{a, \lambda b\})]^{1-\alpha}} f(\max \{a, \lambda b\}) d \lambda \\
& \quad=\int_{0}^{\frac{a}{b}} \frac{(a)^{\prime}}{a[\ln b-\ln a]^{1-\alpha}} f(a) d \lambda+\int_{\frac{a}{b}}^{1} \frac{(\lambda b)^{\prime}}{\lambda b[\ln b-\ln \lambda b]^{1-\alpha}} f(\lambda b) d \lambda \\
& \quad=\int_{a}^{b} \frac{f(t)}{t\left[\ln \frac{b}{t}\right]^{1-\alpha}} d t=\Gamma(\alpha) \mathbf{J}_{a^{+}}^{\alpha} f(b) .
\end{aligned}
$$

If we introduce the right-hand side of the inequality, we come across two cases of $\frac{f(a)}{f(b)}$. One of these cases is $1 \leq \frac{f(a)}{f(b)}$, and in this situation it is

$$
\begin{aligned}
& \int_{0}^{1} \frac{(\max \{a, \lambda b\})^{\prime}}{\max \{a, \lambda b\}[\ln b-\ln (\max \{a, \lambda b\})]^{1-\alpha}} \max \{f(a), \lambda f(b)\} d \lambda \\
& \quad=\int_{0}^{1} \frac{(\max \{a, \lambda b\})^{\prime}}{\max \{a, \lambda b\}[\ln b-\ln (\max \{a, \lambda b\})]^{1-\alpha}} f(a) d \lambda \\
& \quad=f(a)\left[\int_{0}^{\frac{a}{b}} \frac{(a)^{\prime}}{a[\ln b-\ln a]^{1-\alpha}} d \lambda+\int_{\frac{a}{b}}^{1} \frac{(\lambda b)^{\prime}}{\lambda b[\ln b-\ln \lambda b]^{1-\alpha}} d \lambda\right] \\
& \quad=f(a) \int_{\frac{a}{b}}^{1} \frac{1}{\lambda}\left(\ln \frac{1}{\lambda}\right)^{\alpha-1} d \lambda=\frac{f(a)\left(\ln \frac{b}{a}\right)^{\alpha}}{\alpha} .
\end{aligned}
$$

Thence, with these calculations the first part of requested inequality is

$$
\mathbf{J}_{a^{+}}^{\alpha} f(b) \leq \frac{f(a)\left(\ln \frac{b}{a}\right)^{\alpha}}{\Gamma(\alpha+1)}
$$

The other case is $0 \leq \frac{f(a)}{f(b)}<1$ and it must be analyzed in the following separations:

(i) Let $\frac{a}{b} \leq \frac{f(a)}{f(b)}<1$. We attain the following:

$$
\begin{aligned}
\int_{0}^{1} & \frac{(\max \{a, \lambda b\})^{\prime}}{\max \{a, \lambda b\}[\ln b-\ln (\max \{a, \lambda b\})]^{1-\alpha}} \max \{f(a), \lambda f(b)\} d \lambda \\
= & \int_{0}^{\frac{a}{b}} \frac{(a)^{\prime}}{a[\ln b-\ln a]^{1-\alpha}} f(a) d \lambda+\int_{\frac{a}{b}}^{\frac{f(a)}{f(b)}} \frac{(\lambda b)^{\prime}}{\lambda b[\ln b-\ln \lambda b]^{1-\alpha}} f(a) d \lambda \\
& +\int_{\frac{f(a)}{f(b)}}^{1} \frac{(\lambda b)^{\prime}}{\lambda b[\ln b-\ln \lambda b]^{1-\alpha}} \lambda f(b) d \lambda \\
= & f(a) \int_{\frac{a}{b}}^{\frac{f(a)}{f(b)}} \frac{1}{\lambda}\left(\ln \frac{1}{\lambda}\right)^{\alpha-1} d \lambda+f(b) \int_{\frac{f(a)}{f(b)}}^{1}\left(\ln \frac{1}{\lambda}\right)^{\alpha-1} d \lambda \\
= & \frac{f(a)\left[\left(\ln \frac{b}{a}\right)^{\alpha}-\left(\ln \frac{f(b)}{f(a)}\right)^{\alpha}\right]}{\alpha}+f(b) \int_{\frac{f(a)}{f(b)}}^{1}\left(\ln \frac{1}{\lambda}\right)^{\alpha-1} d \lambda .
\end{aligned}
$$


Consequently, we obtain that

$$
\mathbf{J}_{a^{+}}^{\alpha} f(b) \leq \frac{f(a)\left[\left(\ln \frac{b}{a}\right)^{\alpha}-\left(\ln \frac{f(b)}{f(a)}\right)^{\alpha}\right]}{\Gamma(\alpha+1)}+\frac{f(b)}{\Gamma(\alpha)} \int_{\frac{f(a)}{f(b)}}^{1}\left(\ln \frac{1}{\lambda}\right)^{\alpha-1} d \lambda
$$

(ii) For $0 \leq \frac{f(a)}{f(b)}<\frac{a}{b}$, we get that

$$
\begin{aligned}
\int_{0}^{1} & \frac{(\max \{a, \lambda b\})^{\prime}}{\max \{a, \lambda b\}[\ln b-\ln (\max \{a, \lambda b\})]^{1-\alpha}} \max \{f(a), \lambda f(b)\} d \lambda \\
= & \int_{0}^{\frac{f(a)}{f(b)}} \frac{(a)^{\prime}}{a[\ln b-\ln a]^{1-\alpha}} f(a) d \lambda+\int_{\frac{f(a)}{f(b)}}^{\frac{a}{b}} \frac{(a)^{\prime}}{a[\ln b-\ln a]^{1-\alpha}} \lambda f(b) d \lambda \\
& +\int_{\frac{a}{b}}^{1} \frac{(\lambda b)^{\prime}}{\lambda b[\ln b-\ln \lambda b]^{1-\alpha}} \lambda f(b) d \lambda \\
= & f(b) \int_{\frac{a}{b}}^{1}\left(\ln \frac{1}{\lambda}\right)^{\alpha-1} d \lambda .
\end{aligned}
$$

Finally, the inequality is of the following shape:

$$
\mathbf{J}_{a^{+}}^{\alpha} f(b) \leq \frac{f(b)}{\Gamma(\alpha)} \int_{\frac{a}{b}}^{1}\left(\ln \frac{1}{\lambda}\right)^{\alpha-1} d \lambda
$$

Hence, inequality (15) can be derived from (16), (17), and (18).

Theorem 7 Let $\alpha>0$. Iff is a $\mathbb{B}$-convex function on $[a, b]$, then

$$
\mathbf{J}_{b}^{\alpha} f(a) \leq \begin{cases}\frac{f(a)\left(\ln \frac{b}{a}\right)^{\alpha}}{\Gamma(\alpha+1)}, & 1 \leq \frac{f(a)}{f(b)}, \\ \frac{f(a)\left(\ln \frac{b f(a)}{a f(b)}\right)^{\alpha}}{\Gamma(\alpha+1)}+\frac{f(b)}{\Gamma(\alpha)} \int_{\frac{f(a)}{f(b)}}^{1}\left(\ln \frac{\lambda b}{a}\right)^{(\alpha-1)} d \lambda, & \frac{a}{b} \leq \frac{f(a)}{f(b)}<1, \\ \frac{f(b)}{\Gamma(\alpha)} \int_{\frac{a}{b}}^{1}\left(\ln \frac{\lambda b}{a}\right)^{(\alpha-1)} d \lambda, & 0 \leq \frac{f(a)}{f(b)}<\frac{a}{b} .\end{cases}
$$

Proof Since the function $f$ is $\mathbb{B}$-convex, inequality (7) holds. Now, we have to multiply by $\frac{(\max \{a, \lambda b\})^{\prime}}{\max \{a, \lambda b\}[\ln \lambda b-\ln a]^{1-\alpha}}$ and integrate with respect to $\lambda$ over $[0,1]$ to this inequality. Hence, we have

$$
\begin{aligned}
& \int_{0}^{1} \frac{(\max \{a, \lambda b\})^{\prime}}{\max \{a, \lambda b\}[\ln \lambda b-\ln a]^{1-\alpha}} f(\max \{a, \lambda b\}) d \lambda \\
& \quad=\int_{0}^{\frac{a}{b}} \frac{(a)^{\prime}}{a[\ln \lambda b-\ln a]^{1-\alpha}} f(a) d \lambda+\int_{\frac{a}{b}}^{1} \frac{(\lambda b)^{\prime}}{\lambda b[\ln \lambda b-\ln a]^{1-\alpha}} f(\lambda b) d \lambda \\
& \quad=\int_{a}^{b} \frac{f(t)}{t\left[\ln \frac{t}{a}\right]^{1-\alpha}} d t=\Gamma(\alpha) \mathbf{J}_{b^{-}}^{\alpha} f(a) .
\end{aligned}
$$


For the right-hand side of inequality, there are the following two situations that have to be examined. The first is $1 \leq \frac{f(a)}{f(b)}$. Thus,

$$
\begin{aligned}
& \int_{0}^{1} \frac{(\max \{a, \lambda b\})^{\prime}}{\max \{a, \lambda b\}[\ln \lambda b-\ln a]^{1-\alpha}} \max \{f(a), \lambda f(b)\} d \lambda \\
& \quad=\int_{0}^{1} \frac{(\max \{a, \lambda b\})^{\prime}}{\max \{a, \lambda b\}[\ln \lambda b-\ln a]^{1-\alpha}} f(a) d \lambda \\
& \quad=f(a)\left[\int_{0}^{\frac{a}{b}} \frac{(a)^{\prime}}{a[\ln \lambda b-\ln a]^{1-\alpha}} d \lambda+\int_{\frac{a}{b}}^{1} \frac{(\lambda b)^{\prime}}{\lambda b[\ln \lambda b-\ln a]^{1-\alpha}} d \lambda\right] \\
& \quad=f(a) \int_{\frac{a}{b}}^{1} \frac{1}{\lambda}\left(\ln \frac{\lambda b}{a}\right)^{\alpha-1} d \lambda=\frac{f(a)\left(\ln \frac{b}{a}\right)^{\alpha}}{\alpha} .
\end{aligned}
$$

So, the inequality is in the following form:

$$
\mathbf{J}_{b^{-}}^{\alpha} f(a) \leq \frac{f(a)\left(\ln \frac{b}{a}\right)^{\alpha}}{\Gamma(\alpha+1)}
$$

The second case is $0 \leq \frac{f(a)}{f(b)}<1$, and it should be introduced with the following two cases:

(i) One of the cases is $\frac{a}{b} \leq \frac{f(a)}{f(b)}<1$. At this stage, we get that

$$
\begin{aligned}
\int_{0}^{1} & \frac{(\max \{a, \lambda b\})^{\prime}}{\max \{a, \lambda b\}[\ln \lambda b-\ln a]^{1-\alpha}} \max \{f(a), \lambda f(b)\} d \lambda \\
= & \int_{0}^{\frac{a}{b}} \frac{(a)^{\prime}}{a[\ln \lambda b-\ln a]^{1-\alpha}} f(a) d \lambda+\int_{\frac{a}{b}}^{\frac{f(a)}{f(b)}} \frac{(\lambda b)^{\prime}}{\lambda b[\ln \lambda b-\ln a]^{1-\alpha}} f(a) d \lambda \\
& +\int_{\frac{f(a)}{f(b)}}^{1} \frac{(\lambda b)^{\prime}}{\lambda b[\ln \lambda b-\ln a]^{1-\alpha}} \lambda f(b) d \lambda \\
= & f(a) \int_{\frac{a}{b}}^{\frac{f(a)}{f(b)}} \frac{1}{\lambda}\left(\ln \frac{\lambda b}{a}\right)^{\alpha-1} d \lambda+f(b) \int_{\frac{f(a)}{f(b)}}^{1}\left(\ln \frac{\lambda b}{a}\right)^{\alpha-1} d \lambda \\
= & \frac{f(a)\left(\ln \frac{b f(a)}{a f(b)}\right)^{\alpha}}{\alpha}+f(b) \int_{\frac{f(a)}{f(b)}}^{1}\left(\ln \frac{\lambda b}{a}\right)^{\alpha-1} d \lambda .
\end{aligned}
$$

Therefore, the inequality is obtained

$$
\mathbf{J}_{b^{-}}^{\alpha} f(a) \leq \frac{f(a)\left(\ln \frac{b f(a)}{a f(b)}\right)^{\alpha}}{\Gamma(\alpha+1)}+\frac{f(b)}{\Gamma(\alpha)} \int_{\frac{f(a)}{f(b)}}^{1}\left(\ln \frac{\lambda b}{a}\right)^{\alpha-1} d \lambda
$$

(ii) The other one of the cases is $0 \leq \frac{f(a)}{f(b)}<\frac{a}{b}$. So, we have

$$
\begin{aligned}
& \int_{0}^{1} \frac{(\max \{a, \lambda b\})^{\prime}}{\max \{a, \lambda b\}[\ln \lambda b-\ln a]^{1-\alpha}} \max \{f(a), \lambda f(b)\} d \lambda \\
& \quad=\int_{0}^{\frac{f(a)}{f(b)}} \frac{(a)^{\prime}}{a[\ln \lambda b-\ln a]^{1-\alpha}} f(a) d \lambda+\int_{\frac{f(a)}{f(b)}}^{\frac{a}{b}} \frac{(a)^{\prime}}{a[\ln \lambda b-\ln a]^{1-\alpha}} \lambda f(b) d \lambda
\end{aligned}
$$




$$
\begin{aligned}
& +\int_{\frac{a}{b}}^{1} \frac{(\lambda b)^{\prime}}{\lambda b[\ln \lambda b-\ln a]^{1-\alpha}} \lambda f(b) d \lambda \\
= & f(b) \int_{\frac{a}{b}}^{1}\left(\ln \frac{\lambda b}{a}\right)^{\alpha-1} d \lambda .
\end{aligned}
$$

Thus, we get that

$$
\mathbf{J}_{b}^{\alpha} f(a) \leq \frac{f(b)}{\Gamma(\alpha)} \int_{\frac{a}{b}}^{1}\left(\ln \frac{\lambda b}{a}\right)^{\alpha-1} d \lambda
$$

As a result, we have proven inequality (19) by using inequalities (20), (21), and (22).

\subsection{Hermite-Hadamard type inequalities involving fractional integral with respect to the function $g$}

Theorem 8 Let $\alpha>0$ and $0 \leq a<b<+\infty, g:[a, b] \rightarrow \mathbb{R}$ be an increasing and positive monotone function on $(a, b]$, having a continuous derivative $g^{\prime}(x)$ on $(a, b)$. Iff $:[a, b] \rightarrow \mathbb{R}_{+}$ is a $\mathbb{B}$-convex function and $f \in L_{1}[a, b]$, then

$$
\begin{aligned}
& I_{a^{+} ; g}^{\alpha} f(b) \\
& \quad \leq \begin{cases}\frac{f(a)[g(b)-g(a)]^{\alpha}}{\Gamma(\alpha+1)}, & 1 \leq \frac{f(a)}{f(b)}, \\
\frac{\left.f(a)[g(b)-g(a)]^{\alpha}-\left[g(b)-g\left(\frac{b f(a)}{f(b)}\right)\right]^{\alpha}\right]}{\Gamma(\alpha+1)}+\frac{b f(b)}{\Gamma(\alpha)} \int_{\frac{f(a)}{f(b)}}^{1} \frac{g^{\prime}(\lambda b) \lambda}{[g(b)-g(\lambda b)]^{1-\alpha}} d \lambda, & \frac{a}{b} \leq \frac{f(a)}{f(b)}<1, \\
\frac{b f(b)}{\Gamma(\alpha)} \int_{\frac{a}{b}}^{1} \frac{g^{\prime}(\lambda b) \lambda}{[g(b)-g(\lambda b)]^{1-\alpha}} d \lambda, & 0 \leq \frac{f(a)}{f(b)}<\frac{a}{b} .\end{cases}
\end{aligned}
$$

Proof Due to $\mathbb{B}$-convexity of $f:[a, b] \rightarrow \mathbb{R}_{+}$, inequality (7) is valid for all $\lambda \in[0,1]$ and $0<$ $a<b<+\infty$. If we multiply both sides of this inequality by $\frac{g^{\prime}(\max \{a, \lambda b\})}{[g(b)-g(\max \{a, \lambda b\})]^{1-\alpha}}$ and integrate the resulting inequality with respect to $\lambda$ over $[0,1]$, we obtain for the left-hand side of inequality the following equation:

$$
\begin{aligned}
\int_{0}^{1} & \frac{g^{\prime}(\max \{a, \lambda b\})}{[g(b)-g(\max \{a, \lambda b\})]^{1-\alpha}} f(\max \{a, \lambda b\}) d \lambda \\
\quad & \int_{0}^{\frac{a}{b}} \frac{g^{\prime}(a)(a)^{\prime}}{[g(b)-g(a)]^{1-\alpha}} f(a) d \lambda+\int_{\frac{a}{b}}^{1} \frac{g^{\prime}(\lambda b)(\lambda b)^{\prime}}{[g(b)-g(\lambda b)]^{1-\alpha}} f(\lambda b) d \lambda \\
& =\int_{a}^{b} \frac{g^{\prime}(t)}{[g(b)-g(t)]^{1-\alpha}} f(t) d t \\
& =\Gamma(\alpha) I_{a^{+} ; g}^{\alpha} f(b) .
\end{aligned}
$$

For the right-hand side of inequality, we meet two possibilities. One of these is the possibility of $1 \leq \frac{f(a)}{f(b)}$. In this case, the equality is

$$
\begin{gathered}
\int_{0}^{1} \frac{g^{\prime}(\max \{a, \lambda b\})}{[g(b)-g(\max \{a, \lambda b\})]^{1-\alpha}} \max \{f(a), \lambda f(b)\} d \lambda \\
\quad=\int_{0}^{1} \frac{g^{\prime}(\max \{a, \lambda b\})}{[g(b)-g(\max \{a, \lambda b\})]^{1-\alpha}} f(a) d \lambda
\end{gathered}
$$




$$
\begin{aligned}
& =f(a)\left[\int_{0}^{\frac{a}{b}} \frac{g^{\prime}(a)(a)^{\prime}}{[g(b)-g(a)]^{1-\alpha}} d \lambda+\int_{\frac{a}{b}}^{1} \frac{g^{\prime}(\lambda b)(\lambda b)^{\prime}}{[g(b)-g(\lambda b)]^{1-\alpha}} d \lambda\right] \\
& =f(a) \int_{a}^{b} \frac{g^{\prime}(t)}{[g(b)-g(t)]^{1-\alpha}} d t=f(a) \frac{[g(b)-g(a)]^{\alpha}}{\alpha} .
\end{aligned}
$$

Then we deduce that

$$
\begin{aligned}
& \int_{0}^{1} \frac{g^{\prime}(\max \{a, \lambda b\})}{[g(b)-g(\max \{a, \lambda b\})]^{1-\alpha}} f(\max \{a, \lambda b\}) d \lambda \\
& \quad \leq \int_{0}^{1} \frac{g^{\prime}(\max \{a, \lambda b\})}{[g(b)-g(\max \{a, \lambda b\})]^{1-\alpha}} \max \{f(a), \lambda f(b)\} d \lambda, \\
& \Gamma(\alpha) I_{a^{+} ; g}^{\alpha} f(b) \leq f(a) \frac{[g(b)-g(a)]^{\alpha}}{\alpha}, \\
& I_{a^{+} ; g}^{\alpha} f(b) \leq \frac{f(a)[g(b)-g(a)]^{\alpha}}{\Gamma(\alpha+1)} .
\end{aligned}
$$

In the possibility of $0 \leq \frac{f(a)}{f(b)}<1$, we have to examine the following two cases:

(i) $\frac{a}{b} \leq \frac{f(a)}{f(b)}<1$,

(ii) $0 \leq \frac{f(a)}{f(b)}<\frac{a}{b}$.

Let us investigate case (i). If $\frac{a}{b} \leq \frac{f(a)}{f(b)}<1$, then we have

$$
\begin{aligned}
\int_{0}^{1} & \frac{g^{\prime}(\max \{a, \lambda b\})}{[g(b)-g(\max \{a, \lambda b\})]^{1-\alpha}} \max \{f(a), \lambda f(b)\} d \lambda \\
= & \int_{0}^{\frac{a}{b}} \frac{g^{\prime}(a)(a)^{\prime}}{[g(b)-g(a)]^{1-\alpha}} f(a) d \lambda+\int_{\frac{a}{b}}^{\frac{f(a)}{f(b)}} \frac{g^{\prime}(\lambda b)(\lambda b)^{\prime}}{[g(b)-g(\lambda b)]^{1-\alpha}} f(a) d \lambda \\
& +\int_{\frac{f(a)}{f(b)}}^{1} \frac{g^{\prime}(\lambda b)(\lambda b)^{\prime}}{[g(b)-g(\lambda b)]^{1-\alpha}} \lambda f(b) d \lambda \\
= & \frac{f(a)\left[[g(b)-g(a)]^{\alpha}-\left[g(b)-g\left(\frac{b f(a)}{f(b)}\right)\right]^{\alpha}\right]}{\alpha} \\
& +b f(b) \int_{\frac{f(a)}{f(b)}}^{1} \frac{g^{\prime}(\lambda b) \lambda}{[g(b)-g(\lambda b)]^{1-\alpha}} d \lambda .
\end{aligned}
$$

Therefore, we deduce that

$$
I_{a^{+} ; g}^{\alpha} f(b) \leq \frac{f(a)\left[[g(b)-g(a)]^{\alpha}-\left[g(b)-g\left(\frac{b f(a)}{f(b)}\right)\right]^{\alpha}\right]}{\Gamma(\alpha+1)}+\frac{b f(b)}{\Gamma(\alpha)} \int_{\frac{f(a)}{f(b)}}^{1} \frac{g^{\prime}(\lambda b) \lambda}{[g(b)-g(\lambda b)]^{1-\alpha}} d \lambda .
$$

In the case of (ii), $0 \leq \frac{f(a)}{f(b)}<\frac{a}{b}$, we get

$$
\begin{aligned}
& \int_{0}^{1} \frac{g^{\prime}(\max \{a, \lambda b\})}{[g(b)-g(\max \{a, \lambda b\})]^{1-\alpha}} \max \{f(a), \lambda f(b)\} d \lambda \\
& \quad=\int_{0}^{\frac{f(a)}{f(b)}} \frac{g^{\prime}(a)(a)^{\prime}}{[g(b)-g(a)]^{1-\alpha}} f(a) d \lambda+\int_{\frac{f(a)}{f(b)}}^{\frac{a}{b}} \frac{g^{\prime}(a)(a)^{\prime}}{[g(b)-g(a)]^{1-\alpha}} \lambda f(b) d \lambda
\end{aligned}
$$




$$
\begin{aligned}
& +\int_{\frac{a}{b}}^{1} \frac{g^{\prime}(\lambda b)(\lambda b)^{\prime}}{[g(b)-g(\lambda b)]^{1-\alpha}} \lambda f(b) d \lambda \\
= & b f(b) \int_{\frac{a}{b}}^{1} \frac{g^{\prime}(\lambda b) \lambda}{[g(b)-g(\lambda b)]^{1-\alpha}} d \lambda .
\end{aligned}
$$

Consequently, for this case, we obtain the following inequality:

$$
I_{a^{+} ; g}^{\alpha} f(b) \leq \frac{b f(b)}{\Gamma(\alpha)} \int_{\frac{a}{b}}^{1} \frac{g^{\prime}(\lambda b) \lambda}{[g(b)-g(\lambda b)]^{1-\alpha}} d \lambda .
$$

Theorem 9 Let $\alpha>0$ and $0<a<b<+\infty, g:[a, b] \rightarrow \mathbb{R}$ be an increasing and positive monotone function on $(a, b]$, having a continuous derivative $g^{\prime}(x)$ on $(a, b)$. Iff $:[a, b] \rightarrow \mathbb{R}_{+}$ is a $\mathbb{B}$-convex function and $f \in L_{1}[a, b]$, then the following inequality for fractional integrals holds:

$$
I_{b^{-} ; g}^{\alpha} f(a) \leq \begin{cases}\frac{f(a)[g(b)-g(a)]^{\alpha}}{\Gamma(\alpha+1)}, & 1 \leq \frac{f(a)}{f(b)}, \\ \frac{f(a)\left[g\left(\frac{b f(a)}{f(b)}\right)-g(a)\right]^{\alpha}}{\Gamma(\alpha+1)}+\frac{b f(b)}{\Gamma(\alpha)} \int_{\frac{f(a)}{f(b)}}^{1} \frac{g^{\prime}(\lambda b) \lambda}{[g(\lambda b)-g(a)]^{1-\alpha}} d \lambda, & \frac{a}{b} \leq \frac{f(a)}{f(b)}<1, \\ \frac{b f(b)}{\Gamma(\alpha)} \int_{\frac{a}{b}}^{1} \frac{g^{\prime}(\lambda b) \lambda}{[g(\lambda b)-g(a)]^{1-\alpha}} d \lambda, & 0 \leq \frac{f(a)}{f(b)}<\frac{a}{b} .\end{cases}
$$

Proof For the $\mathbb{B}$-convex function $f:[a, b] \rightarrow \mathbb{R}_{+}$, we have the following inequality:

$$
f(\max \{a, \lambda b\}) \leq \max \{f(a), \lambda f(b)\}
$$

for all $\lambda \in[0,1]$. To obtain inequality (24), we should multiply both sides of (25) by $\frac{g^{\prime}(\max \{a, \lambda b])}{[g(\lambda b)-g(a)]^{1-\alpha}}$ and integrate the resulting inequality with respect to $\lambda$ over $[0,1]$. Thus, for the left-hand side of inequality, we get

$$
\begin{aligned}
& \int_{0}^{1} \frac{g^{\prime}(\max \{a, \lambda b\})}{[g(\lambda b)-g(a)]^{1-\alpha}} f(\max \{a, \lambda b\}) d \lambda \\
& \quad=\int_{\frac{a}{b}}^{1} \frac{g^{\prime}(\lambda b)(\lambda b)^{\prime}}{[g(\lambda b)-g(a)]^{1-\alpha}} f(\lambda b) d \lambda \\
& \quad=\int_{a}^{b} \frac{g^{\prime}(t)}{[g(t)-g(a)]^{1-\alpha}} f(t) d t=\Gamma(\alpha) I_{b^{-;} ; g}^{\alpha} f(a) .
\end{aligned}
$$

For the right-hand side of inequality, we have to consider two cases as follows. Firstly, it can be $1 \leq \frac{f(a)}{f(b)}$. In this case, for the right-hand side, we obtain that

$$
\begin{aligned}
\int_{0}^{1} \frac{g^{\prime}(\max \{a, \lambda b\})}{[g(\lambda b)-g(a)]^{1-\alpha}} \max \{f(a), \lambda f(b)\} d \lambda & =\int_{\frac{a}{b}}^{1} \frac{g^{\prime}(\lambda b)(\lambda b)^{\prime}}{[g(\lambda b)-g(a)]^{1-\alpha}} f(a) d \lambda \\
& =\frac{f(a)[g(b)-g(a)]^{\alpha}}{\alpha} .
\end{aligned}
$$

Hence,

$$
\begin{aligned}
& \Gamma(\alpha) I_{b^{-} ; g}^{\alpha} f(a) \leq \frac{f(a)[g(b)-g(a)]^{\alpha}}{\alpha}, \\
& I_{b^{-} ; g}^{\alpha} f(a) \leq \frac{f(a)[g(b)-g(a)]^{\alpha}}{\Gamma(\alpha+1)} .
\end{aligned}
$$


When we handle the second case $0 \leq \frac{f(a)}{f(b)}<1$, we meet the following two possibilities:

(i) $\frac{a}{b} \leq \frac{f(a)}{f(b)}<1$,

(ii) $0 \leq \frac{f(a)}{f(b)}<\frac{a}{b}$.

For (i), let us calculate the right-hand side of inequality and then show the last case.

$$
\begin{aligned}
& \int_{0}^{1} \frac{g^{\prime}(\max \{a, \lambda b\})}{[g(\lambda b)-g(a)]^{1-\alpha}} \max \{f(a), \lambda f(b)\} d \lambda \\
& \quad=\int_{\frac{a}{b}}^{\frac{f(a)}{f(b)}} \frac{g^{\prime}(\lambda b)(\lambda b)^{\prime}}{[g(\lambda b)-g(a)]^{1-\alpha}} f(a) d \lambda+\int_{\frac{f(a)}{f(b)}}^{1} \frac{g^{\prime}(\lambda b)(\lambda b)^{\prime}}{[g(\lambda b)-g(a)]^{1-\alpha}} \lambda f(b) d \lambda \\
& \quad=\frac{f(a)\left[g\left(\frac{b f(a)}{f(b)}\right)-g(a)\right]^{\alpha}}{\alpha}+b f(b) \int_{\frac{f(a)}{f(b)}}^{1} \frac{g^{\prime}(\lambda b) \lambda}{[g(\lambda b)-g(a)]^{1-\alpha}} d \lambda .
\end{aligned}
$$

Thus, we have that

$$
\begin{aligned}
& \Gamma(\alpha) I_{b^{-} ; g}^{\alpha} f(a) \leq \frac{f(a)\left[g\left(\frac{b f(a)}{f(b)}\right)-g(a)\right]^{\alpha}}{\alpha}+b f(b) \int_{\frac{f(a)}{f(b)}}^{1} \frac{g^{\prime}(\lambda b) \lambda}{[g(\lambda b)-g(a)]^{1-\alpha}} d \lambda, \\
& I_{b^{-} ; g}^{\alpha} f(a) \leq \frac{f(a)\left[g\left(\frac{b f(a)}{f(b)}\right)-g(a)\right]^{\alpha}}{\Gamma(\alpha+1)}+\frac{b f(b)}{\Gamma(\alpha)} \int_{\frac{f(a)}{f(b)}}^{1} \frac{g^{\prime}(\lambda b) \lambda}{[g(\lambda b)-g(a)]^{1-\alpha}} d \lambda .
\end{aligned}
$$

For case (ii), we have that

$$
\begin{aligned}
\int_{0}^{1} \frac{g^{\prime}(\max \{a, \lambda b\})}{[g(\lambda b)-g(a)]^{1-\alpha}} \max \{f(a), \lambda f(b)\} d \lambda & =\int_{\frac{a}{b}}^{1} \frac{g^{\prime}(\lambda b)(\lambda b)^{\prime}}{[g(\lambda b)-g(a)]^{1-\alpha}} \lambda f(b) d \lambda \\
& =b f(b) \int_{\frac{a}{b}}^{1} \frac{g^{\prime}(\lambda b) \lambda}{[g(\lambda b)-g(a)]^{1-\alpha}} d \lambda .
\end{aligned}
$$

Thus, we deduce

$$
\begin{aligned}
& \Gamma(\alpha) I_{b^{-} ; g}^{\alpha} f(a) \leq b f(b) \int_{\frac{a}{b}}^{1} \frac{g^{\prime}(\lambda b) \lambda}{[g(\lambda b)-g(a)]^{1-\alpha}} d \lambda, \\
& I_{b^{-} ; g}^{\alpha} f(a) \leq \frac{b f(b)}{\Gamma(\alpha)} \int_{\frac{a}{b}}^{1} \frac{g^{\prime}(\lambda b) \lambda}{[g(\lambda b)-g(a)]^{1-\alpha}} d \lambda .
\end{aligned}
$$

Consequently, from (26), (27), and (28), we have inequality (24).

The given results in Theorem 8 and Theorem 9 are the most general HermiteHadamard inequalities for $\mathbb{B}$-convex functions.

Corollary 1 Inequalities (23) and (24) are generalized forms of inequalities (11) and (10), respectively.

Indeed, observe that for $g(x)=x$, the fractional integral (5) reduces to the left-sided Riemann-Liouville fractional integral (1), and the fractional integral (6) reduces to the right-sided Riemann-Liouville fractional integral (2). 
Additionally, these hypotheses are valid in our results. Namely, if we get $g(x)=x$ in (23), the inequality returns to (11). Similarly, getting $g(x)=x$ in (24) gives inequality (10).

Corollary 2 Hermite-Hadamard inequality for a $\mathbb{B}$-convex function involving Hadamard fractional integral is obtained from inequalities (23) and (24).

This can be seen by using the same method in Corollary 1.

We can give the following examples for inequalities (23) and (24) to be more precise.

Example 1 Let us assume that $\alpha=2, f:[1,2] \rightarrow \mathbb{R}_{+}, f(x)=x^{2}$, and $g:[1,2] \rightarrow \mathbb{R}_{+}, g(x)=$ $x^{3}$. Indeed the function $f$ satisfies inequality (7), namely it is a $\mathbb{B}$-convex function. Thus, if we examine inequality (23) under all these assumptions, then we deduce

$$
I_{1^{+} ; x^{3}}^{2} f(2)=\frac{1}{\Gamma(2)} \int_{1}^{2} \frac{3 t^{2} \cdot t^{2}}{\left[2^{3}-t^{3}\right]^{1-2}} d t=\frac{2127}{40}<\frac{2 \cdot 2^{2}}{\Gamma(2)} \int_{\frac{1}{2}}^{1} \frac{3(2 \lambda)^{2} \lambda}{\left[2^{3}-(2 \lambda)^{3}\right]^{1-2}} d \lambda=\frac{498}{7}
$$

because of $0 \leq \frac{f(a)}{f(b)}=\frac{1}{4}<\frac{a}{b}=\frac{1}{2}$. Additionally, if inequality (24) is analyzed in the present circumstances, then one has

$$
I_{2^{-} ; x^{3}}^{2} f(1)=\frac{1}{\Gamma(2)} \int_{1}^{2} \frac{3 t^{2} \cdot t^{2}}{\left[t^{3}-1^{3}\right]^{1-2}} d t=\frac{3081}{40}<\frac{2 \cdot 2^{2}}{\Gamma(2)} \int_{\frac{1}{2}}^{1} \frac{3(2 \lambda)^{2} \lambda}{\left[(2 \lambda)^{3}-1^{3}\right]^{1-2}} d \lambda=\frac{1209}{14} .
$$

Furthermore, it can be seen that all inequalities (10), (11), (15), and (19) are valid for the same function.

For more examples of $\mathbb{B}$-convex functions, see [9].

Acknowledgements

The author wishes to thank Aksaray University and TUBITAK (The Scientific and Technological Research Council of Turkey).

Funding

Not applicable.

Availability of data and materials

Not applicable.

Competing interests

The author declares that she has no competing interests.

Authors' contributions

IY completed the work alone. The author read and approved the final manuscript.

Authors' information

IY is an Assistant Professor in the Department of Mathematics at Aksaray University, Aksaray, Turkey.

\section{Publisher's Note}

Springer Nature remains neutral with regard to jurisdictional claims in published maps and institutional affiliations.

Received: 14 February 2018 Accepted: 5 July 2019 Published online: 12 July 2019

References

1. Adilov, G.: Increasing co-radiant functions and Hermite-Hadamard type inequalities. Math. Inequal. Appl. 14(1), 45-60 (2011)

2. Adilov, G., Kemali, S.: Hermite-Hadamard-type inequalities for increasing positively homogeneous functions. J. Inequal. Appl. 2007, 021430 (2007)

3. Adilov, G., Kemali, S.: Abstract convexity and Hermite-Hadamard type inequalities. J. Inequal. Appl. 2009, 943534 (2009) 
4. Adilov, G., Rubinov, A.: $\mathbb{B}$-Convex sets and functions. Numer. Funct. Anal. Optim. 27(3-4), 237-257 (2006)

5. Adilov, G., Tinaztepe, G.: The sharpening of some inequalities via abstract convexity. Math. Inequal. Appl. 12(1), 33-51 (2009)

6. Adilov, G., Yesilce, I.: $\mathbb{B}^{-1}$-Convex sets and $\mathbb{B}^{-1}$-measurable maps. Numer. Funct. Anal. Optim. 33(2), 131-141 (2012)

7. Adilov, G., Yesilce, I.: On generalization of the concept of convexity. Hacet. J. Math. Stat. 41(5), 723-730 (2012)

8. Adilov, G., Yesilce, I.: $\mathbb{B}^{-1}$-Convex functions. J. Convex Anal. 24(2), 505-517 (2017)

9. Adilov, G., Yesilce, I.: Some important properties of $\mathbb{B}$-convex function. J. Nonlinear Convex Anal. 19(4), 669-680 (2018)

10. Agarwal, P.: Further results on fractional calculus of Saigo operators. Appl. Appl. Math. 7(2), 585-594 (2012)

11. Agarwal, P.: Some inequalities involving Hadamard-type k-fractional integral operators. Math. Methods Appl. Sci. 40(11), 3882-3891 (2017)

12. Agarwal, P., Al-Mdallal, Q., Cho, Y.J., Jain, S.: Fractional differential equations for the generalized Mittag-Leffler function. Adv. Differ. Equ. 2018, 58 (2018)

13. Agarwal, P., Dragomir, S.S., Jleli, M., Samet, B.: Advances in Mathematical Inequalities and Applications. Springer, Berlin (2018)

14. Agarwal, P., Jeli, M., Tomar, M.: Certain Hermite-Hadamard type inequalities via generalized k-fractional integrals. J. Inequal. Appl. 2017, 55 (2017)

15. Agarwal, P., Nieto, J..: Some fractional integral formulas for the Mittag-Leffler type function with four parameters. Open Math. 13(1), 537-546 (2015)

16. Briec, W., Horvath, C.D.: $\mathbb{B}$-Convexity. Optimization 53(2), 107-127 (2004)

17. Dahmani, Z:: On Minkowski and Hermite-Hadamard integral inequalities via fractional integration. Ann. Funct. Anal. 1(1), 51-58 (2010)

18. Dragomir, S.S., Pearce, C.E.M.: Selected topics on Hermite-Hadamard inequalities and applications (2000)

19. Hadamard, J.: Etude sur les proprietes des fonctions entieres et en particulier d'une fonction consideree par Riemann. J. Math. Pures Appl. 58, 171-215 (1893)

20. Hermite, C.H.: Sur deux limites d'une integrale define. Mathesis 3, 82 (1883)

21. Jain, S., Agarwal, P., Kiymaz, I.O., Cetinkaya, A.: Some composition formulae for the MSM fractional integral operator with the multi-index Mittag-Leffler functions. AIP Conf. Proc. 1926(1), 020020 (2018)

22. Jain, S., Mehrez, K., Baleanu, D., Agarwal, P.: Certain Hermite-Hadamard inequalities for logarithmically convex functions with applications. Mathematics 7(2), 163 (2019)

23. Kemali, S., Yesilce, I., Adilov, G.: $\mathbb{B}$-Convexity, $\mathbb{B}^{-1}$-convexity, and their comparison. Numer. Funct. Anal. Optim. 36(2), 133-146 (2015)

24. Kilbas, A.A., Marichev, O.I., Samko, S.G.: Fractional Integrals and Derivatives. Theory and Applications. Gordon \& Breach, Switzerland (1993)

25. Kilbas, A.A., Srivastava, H.M., Trujillo, J.J.: Theory and Applications of Fractional Differential Equations. Elsevier, Amsterdam (2006)

26. Liouville, J.: Memoire sur le calcul des differentielles a indices quelconques. J. Éc. Polytech. 13(21), 71-162 (1832)

27. Mehrez, K., Agarwal, P.: New Hermite-Hadamard type integral inequalities for convex functions and their applications. J. Comput. Appl. Math. 350, 274-285 (2019)

28. Niculescu, C.P., Persson, L.E.: Old and new on the Hermite-Hadamard inequality. Real Anal. Exch. 29(2), 663-685 (2003)

29. Oldham, K.B., Spanier, J.: The Fractional Calculus. Theory and Applications of Differentiation and Integration of Arbitrary Order. Dower, New York (2006)

30. Riemann, B.: Versuch einer allgemeinen Auffasung der Integration und Differentiation. In: Weber, H. (ed.) Bernhard Riemanns gesammelte mathematische Werke und wissenschaftlicher Nachlass. Dover, New York (1953), 353

31. Ruzhansky, M.V., Je Cho, Y., Agarwal, P., Area, I.: Advances in Real and Complex Analysis with Applications. Springer, Singapore (2017)

32. Sarikaya, M.Z., Set, E., Yaldiz, H., Basak, N.: Hermite-Hadamard's inequalities for fractional integrals and related fractional inequalities. Math. Comput. Model. 57(9), 2403-2407 (2013)

33. Sitho, S., Ntouyas, S.K., Agarwal, P., Tariboon, J: Noninstantaneous impulsive inequalities via conformable fractional calculus. J. Inequal. Appl. 2018, 261 (2018)

34. Tinaztepe, G., Yesilce, I., Adilov, G.: Separation of $\mathbb{B}^{-1}$-convex sets by $\mathbb{B}^{-1}$-measurable maps. J. Convex Anal. 21(2), 571-580 (2014)

35. Yesilce, I., Adilov, G.: Hermite-Hadamard inequalities for $L(j)$-convex functions and $S(j)$-convex functions. Malaya J. Mat. 3(3), 346-359 (2015)

36. Yesilce, I., Adilov, G.: Fractional integral inequalities for $\mathbb{B}$-convex functions. Creative Math. Inform. 26(3), 345-351 (2017)

37. Yesilce, I., Adilov, G.: Hermite-Hadamard inequalities for $\mathbb{B}$-convex and $\mathbb{B}^{-1}$-convex functions. Int. J. Nonlinear Anal. Appl. 8(1), 225-233 (2017) 